\title{
Intención sucesoria de los estudiantes de escuelas agrarias en las agroindustrias familiares
}

\author{
Manel Plana Farran ${ }^{\text {a* }}$ y José Luis Gallizo Larraz ${ }^{\text {b }}$ \\ a Profesor asociado en el departamento de Administración de Empresas de la Universidad de Lleida \\ ${ }^{\mathrm{b}}$ Director de la Cátedra de Empresa Familiar de la Universidad de Lleida \\ *Correo de contacto: manel.plana@udl.cat
}

Recibido 30 de noviembre de 2020; Aceptado 13 de diciembre de 2020

\begin{abstract}
Resumen
Este trabajo aborda la problemática de la sucesión en las explotaciones agrarias familiares desde la perspectiva del individuo que debe tomar una decisión vital para orientar su futuro profesional sobre la base de sus capacidades, expectativas y emociones. Mediante una encuesta dirigida a los asistentes a las escuelas agrarias de Cataluña nuestro estudio pretende averiguar cuáles son los factores determinantes de la sucesión intergeneracional de las explotaciones agrarias familiares. Hemos obtenido una validación empírica que relaciona la intención sucesoria en la EAF y la riqueza socioemocional de las familias empresarias a través de un análisis factorial. Los resultados muestran que además de las características del individuo, la intención de administrar la finca aumenta con el interés del propio individuo de crear su propio negocio y de la inclinación emocional de continuar con el legado familiar.
\end{abstract}

Clasificación JEL: Q15,

Palabras Clave: Explotaciones agrarias familiares, sucesión, riqueza socioemecional.

\section{Introducción}

La consecución de supervivencia de la explotación agraria familiar (EAF) está condicionada por la capacidad de designar un sucesor o heredero de la finca familiar (Iinwood y Sharp, 2012; Mann et al., 2013). La actividad agrícola en una finca difícilmente será asumida por alguien ajeno a la familia que compre la explotación, dadas las altas inversiones necesarias y la baja rentabilidad que se espera, además, la mano de obra familiar reduce los costos de producción en comparación con la mano de obra contratada. Un inversor externo quedaría por si mismo descartado en la compra de la finca, salvo que tuviera proximidad de sus medios de producción que permitiera obtener economías de escala con la adquisición de una nueva propiedad. (Cavichiolli et al. (2015). 
Por la dificultad que entraña la transmisión de la propiedad, no es de extrañar que el proceso de sucesión sea una preocupación entre los empresarios rurales y clave para la supervivencia de las empresas agrícolas familiares (Lange et al., 2013).

Para ayudar a planificar el proceso sucesorio, diferentes administraciones públicas están desarrollando estrategias de apoyo a los sucesores en obtener una mayor concienciación sobre los vínculos entre las cuestiones relacionadas con la sucesión, la longevidad empresarial y el aumento de la competitividad (Suess-Reyes y Fuetsch, 2016).

El lugar donde estas estrategias tienen mayor capacidad de arraigo es en las escuelas agrarias, denominadas en España, Escuelas de Capacitación Agraria y agrupadas en Europa en la Red de Escuelas "Europea-International" (www.europea.org) que lleva a cabo proyectos de innovación pedagógica, de intercambio de estudiantes y profesores y organiza competiciones y concursos de estudiantes.

Entre los objetivos de las escuelas agrarias está el de favorecer las incorporaciones de jóvenes al campo formándoles para garantizar la rentabilidad de las explotaciones, recuperando la autoestima de los agricultores y explicando a la sociedad la importancia que tiene la producción de alimentos y servicios que proporcionan las empresas agrarias. En estos centros educativos se produce un encuentro entre los jóvenes que han tomado la decisión de dedicar su vida laboral a la agricultura y que en muchos casos forman parte de familias de agricultores. Es aquí donde podemos encontrar las respuestas para explicar los factores que influyen en la sucesión intergeneracional en las familias de agricultores.

Estudios previos han proporcionado información de interés sobre cómo los resultados de la sucesión se ven afectados por estos factores o una combinación de factores, sin embargo, en los mismos se reconoce un valor explicativo bajo, en parte atribuyéndolo a la falta de datos disponibles y a factores no observables (Hennessey y Rehmann, 2007; p. 27).

Normalmente, los académicos ven la sucesión en el campo como un proceso impulsado por factores discretos como el nivel de educación, el tamaño de la finca, la rentabilidad, el tipo de empresa, etc., (Lobley y Potter, 2004). Nuestro trabajo se fundamenta en que la clave de la sucesión está en los individuos que toman una decisión vital para orientar su futuro profesional sobre la base de sus capacidades, expectativas y emociones.

En este trabajo nos hemos dirigido a los asistentes a las escuelas agrarias de Cataluña para realizar un estudio que nos permita averiguar cuales son los factores influyentes en la sucesión intergeneracional en las explotaciones agrarias familiares. La investigación se basa en una encuesta realizada a estudiantes de las Escuelas Agrarias de Cataluña y su mayor valor es la representatividad de la muestra, hemos contado con el 54\% de la población.

A partir de las respuestas obtenidas, se ha realizado un análisis inferencial comparativo sobre la intención sucesoria de la explotación agraria familiar en función de los factores intrínsecos del encuestado y posteriormente, un análisis factorial que nos ha permitido establecer una relación entre la intención sucesoria en la EAF y la riqueza socioemocional de los individuos encuestados pertenecientes a explotaciones agrarias familiares. 
Nuestros resultados implican la preferencia de hombres frente a las mujeres en la selección previa hacia los estudios de agricultura $\mathrm{y}$, por tanto, como potenciales sucesores de las explotaciones. El interés propio es un factor de alta importancia, así, los sucesores potenciales que realizan estudios en agricultura se dan cuenta de su capacidad para controlar la empresa familiar rural. Se ha observado que, a medida que el deseo de trabajar en el sector agrícola aumenta, su intención de administrar la finca también aumenta y el interés por crear su propio negocio también es un factor de interés. Esto indica que aparte de las características de la explotación, la capacidad e inclinación del potencial sucesor de permanecer en la empresa familiar, son factores para considerar respecto a la intención sucesoria de los jóvenes estudiantes de escuelas agrarias. Los resultados son importantes para planificar la sucesión en las familias de agricultores.

El resto del trabajo se organiza de la siguiente forma: En la sección 2 se lleva a cabo una revisión de la literatura previa, en la sección 3 , se explica la muestra de individuaos a los que se dirigió la encuesta y la metodología aplicada. En la sección 4 se describen los resultados del análisis inferencial y en la sección 5, se presentan los resultados obtenidos del estudio que relaciona la intención sucesoria con la riqueza socioemocional de la explotación agraria familiar. Finalmente, la sección 6 se dedica a la discusión y conclusiones del trabajo.

\section{Antecedentes y planteamiento de la investigación}

Las Explotaciones Agrarias Familiares se encuentran expuestas en la actualidad a unos cambios muy severos tanto a nivel económico, como social y ambiental. La seguridad alimentaria, las crecientes exigencias en los estándares de calidad y los cambios en los gustos de los clientes contribuyen al nuevo escenario en el que se ve obligado a actuar el sector agrario (Alsos et al., 2011). La volatilidad en los precios de mercados (Darnhofer et al., 2010), la necesidad de aplicación de nuevas tecnologías a la actividad agraria, el cambio climático con unas consecuencias directas en el modelo productivo hace que el sector agroalimentario se encuentre ante una situación de cambio, desafío y necesidad de adaptación constante que alcanza a todos sus actores, y en especial a los potenciales sucesores de Explotaciones Agrarias Familiares. Todos estos cambios exigen al agricultor actual y a las siguientes generaciones de conocimientos y de formación que complemente el conocimiento practico adquirido en la explotación agraria familiar.

Por otra parte, se constata la disminución progresiva del número de jóvenes que se incorporan a la actividad agraria, cuestión que se atribuye a los cambios tanto a nivel tecnológico, como económico y social (Dinis, 2019; Leonard et al., 2017; Morais et al., 2018). Esta situación genera un envejecimiento progresivo en la edad del agricultor y una falta de relevo generacional en las explotaciones agrarias familiares que queremos analizar.

Chiswell (2014) reclama el análisis de la sucesión en las explotaciones agrarias familiares desde la perspectiva del potencial o futuro sucesor y no como figuras marginales encuadradas en el concepto general de agricultor, condición que se le atribuye, generalmente, al titular de la explotación agraria.

La baja proporción de jóvenes agricultores se ve como un problema tanto por parte de Instituciones gubernamentales y gobiernos de diferentes países como por parte de diferentes actores y organizaciones profesionales agrarias. Zagata y Sutherland (2015) argumentan que la baja proporción de jóvenes en las explotaciones agrarias se percibe 
como una pérdida de potencial a la hora de crear unas explotaciones agrarias más eficientes, competitivas y sostenibles acorde con las exigencias derivadas del entorno y del propio sector.

Con unos precedentes que se remontan a los inicios del S.XX, en el año 1980 se creó por parte de la Generalitat de Catalunya, y dentro del Departament d'Agricultura, Ramaderia i Pesca, el Servicio de Capacitación Agraria, siendo una apuesta importante de fijar la formación agraria en el ámbito del Departament d'Agricultura. En el año 2002 se transformó en el Servei de Formació Agrària y que tiene los siguientes cometidos:

- Ejecutar los planes y los programas de capacitación profesional agraria del Departament, tanto en el ámbito de la formación inicial como en el reciclaje y renovación de conocimientos y la formación permatenete de los agricultores y en general del entorno rural.

- Hacer un seguimiento y evaluación de los planes y los programas de capacitación profesional agraria.

- Supervisar el funcionamiento de las escuelas y los centros de capacitación agraria.

Las escuelas agrarias son nuestro campo de actuación en esta investigación, a partir de ellas obtenemos los datos de jóvenes con un proyecto de incorporación a la actividad agrícola, en la mayoría de los casos, pertenecientes a familias agrarias

\subsection{Factores influyentes en la intención sucesoria}

Aunque en muchas familias no existan reglas de sucesión explícitas. Las decisiones que al final toman las familias incluyen factores vinculados al número de sucesores, su género, el orden de nacimiento, su dedicación y sus habilidades personales para la gestión de la empresa y para el trabajo agropecuario (Keating y Munro, 1989).

Sobre la base de estudios previos hemos identificado factores que influyen en la sucesión intergeneracional de negocios rurales agrícolas como son:

1. El género. El sector agrario es un ámbito altamente masculinizado (Glover, 2014). El género es una de las primeras identidades individuales que entra a formar parte en el proceso de sucesión, que, por ser natural, no es elegible y organiza el comportamiento de formas más categóricas que pueden construirse posteriormente (Jenkins, 1996). Las mujeres no son generalmente percibidas como sucesoras elegibles y más cuando hay hijos varones, sin embargo, desde hace unos cuantos años se está produciendo una corrección de esta tendencia que es necesario comprobar.

2. La edad. La edad del encuestado como potencial sucesor es un elemento que valoramos en la encuesta realizada. Son estudiantes de las escuelas agrarias, con diferencias de edad que pueden ofrecer una perspectiva distinta sobre la expectativa de la sucesión de la explotación familiar. Los de mayor edad pueden ver más próxima su incorporación al campo, mientras que los más jóvenes con intenciones de continuar los estudios pueden opinar que su futuro no terminará en la explotación familiar. 
3. El interés propio. La intención de un sucesor potencial para gestionar el negocio de la empresa familiar agrícola se determinó como un importante factor en trabajos anteriores. Se valoró positivamente la adquisición del negocio familiar rural y se tuvieron percepciones positivas sobre la capacidad para administrar el negocio agrícola familiar y sus percepciones sobre la presión social para controlar la propiedad familiar (Morais et al., 2018). En nuestro trabajo, hemos preguntado a los encuestados sobre su interés particular para continuar con el negocio familiar, entendiendo que este es uno de los principales elementos de decisión, es decir, que el individuo esté decidido a dedicar su vida a la actividad agrícola y de administración de la explotación.

4. El conocimiento informal y formal. El conocimiento informal se obtiene por el aprendizaje que no cuenta con una estructura mediante un programa con inicio y fin, si no que surge de acuerdo con las necesidades contingentes de los usuarios que consultan fuentes para resolver problemas puntuales, o tomar decisiones de forma inmediata. Sumane et al. (2018) argumentan la necesaria complementariedad en el sector agrario del conocimiento formal e informal. Rosenzweig y Wolpin (1985), así como Corsi (2004), que obtuvo que el conocimiento específico de las explotaciones agrarias crea incentivos en los hijos para continuar con la explotación agraria familiar. Se constata que el interés de los hijos por la agricultura es un factor con el que contar y tener en cuenta a la hora de analizar las características de los potenciales y/o futuros sucesores de las explotaciones agrarias familiares (Lobley et al., 2010).

\subsection{Relación entre intención sucesoria y SEW}

Se ha estudiado que las EF limitan el objetivo de maximización del beneficio a cambio de mantener el control de la empresa y de transmitir ese control a futuras generaciones (Gallizo et al., 2017). Las explotaciones agrarias familiares poseen además de la propiedad económica unos valores afectivos que influyen en el proceso de sucesión. Esos valores afectivos de las familias empresarias se han agrupado bajo el concepto de riqueza socioemocional, SEW.

La SEW se define como la "dotación afectiva de los propietarios familiares" (GómezMejía et al., 2011; p. 654), es decir, las utilidades o valores afectivos no económicos que una familia deriva de su posición de propiedad en una determinada empresa. (Berrone et al., 2012; Gómez-Mejía et al., 2007; Gómez-Mejía et al., 2010; GómezMejía et al., 2011).

Tanto los valores económicos como afectivos derivados de la propiedad familiar pueden influir en la toma de decisiones en las empresas familiares.

El porcentaje de propiedad familiar se ha descrito como un "impulsor teórico" de SEW (Schulze y Kellermanns, 2015). En consecuencia, la propiedad familiar en una empresa conduce a valores afectivos multifacéticos y, por lo tanto, diversos para la familia propietaria, que se conceptualizan como SEW.

Otras investigaciones han demostrado que una de las características típicas de las empresas familiares es que una parte importante de su comportamiento está motivado y focalizado en el sostenimiento del SEW, referido a aspectos no financieros o al 
"patrimonio afectivo" de los propietarios familiares (Gómez-Mejía et al. 2011; Naldi et al., 2013).

En este trabajo se ha ampliado el uso y el análisis del SEW a los potenciales sucesores de las explotaciones agrarias familiares, que se están formando mediante un conocimiento reglado y formal en las escuelas agrarias, con el objetivo de incorporarse a la explotación agraria familiar y hacerse cargo de la misma. La intención sucesoria de estos estudiantes viene refrendada por una de las motivaciones principales que les ha llevado a manifestar que quieren continuar con la explotación familiar agraria: "la continuidad de la tradición familiar y el modelo de vida que representa el trabajo en la explotación agraria". Estos aspectos corroboran la existencia de un "patrimonio afectivo" que no solo se encuentra en manos de los propietarios familiares, también de los potenciales y futuros sucesores. Ésto nos lleva a establecer la existencia de una intensa relación entre entre la intención sucesoria del futuro sucesor y el SEW.

Además nos indica la necesidad que ya apuntaba Chiswell (2014) de ampliar y aplicar el análisis del SEW no únicamente a los propietarios, también a otros miembros de la explotación agraria familiar, en especial a los futuros y/o potenciales sucesores.

\section{Muestra y metodología}

Para identificar los factores que determinan la sucesión intergeneracional en las explotaciones agrarias familiares se recopiló información de las 13 Escuelas Agrarias de Cataluña.

Se ha logrado recoger información de 374 participantes. La participación por Escuela (Fig. 1) varía desde un mínimo de 12 alumnos (en la $\mathrm{n}^{\circ}$ 5) hasta un máximo de 78 (en la $\left.\mathrm{n}^{\mathrm{o}} 11\right)$. El número medio de participantes es de casi 29 (28.8) por escuela.

Consideramos que esta muestra de 374 participantes (sobre población de 700) es estadísticamente representativa con un margen de error máximo del $\pm 3.46 \%$, bajo el supuesto de máxima variabilidad $(\mathrm{p}=\mathrm{q}=50 \%)$ y fijando el habitual margen de confianza del $95 \%$.

Con las respuestas de estos 374 participantes se ha configurado un perfil de alumno en base a 3 cuestiones: edad, sexo y tipo de actividad. Se observa que:

- Hay una mayoría de hombres frente a mujeres: $77.8 \%$ vs $22.2 \%$; es decir una ratio 3.5:1, que es mayoría estadísticamente significativa con $\mathrm{p}<.001$ (Test Chicuadrado: valor=115.68; $\mathrm{p}$-valor exacto=.000000). Desde este dato, se estima que la presencia de hombres en los estudios agrarios, al 95\% de confianza, está comprendida entre un $73.2 \%$ y un $81.9 \%$.

- La edad más frecuente se encuentra en la banda de los 15-20 años, es decir los más jóvenes: $71.9 \%$ (IC: $67.1 \%$ - 76.4\%). De este primer grupo, podemos decir que son mayoría significativa con $\mathrm{p}<.001$ (Test Chi-cuadrado: valor $=445.72$; p-valor exacto $=.000000)$. Los que superan esa edad se reparte casi por igual entre el intervalo de $21-25$ años (14.7\%) y los mayores de esa edad (13.3\%).

Y finalmente en cuanto al tipo de explotación, se ha observado que un 43\% (IC: $38.0 \%$ - 48.2\%) de los alumnos, es decir muy cerca de la mitad, están estudiando en estas 
Escuelas procediendo de explotaciones agrarias familiares. Pero también, la otra mitad (45.5; IC: 40.3\% - 50.7\%) estudian en estas escuelas sin tener ninguna vinculación con un negocio agrario. El emprendimiento personal apenas tiene peso $(2.1 \%$; IC: $0.9 \%$ $4.2 \%)$

Figura 1. Procedencia de la muestra según escuela

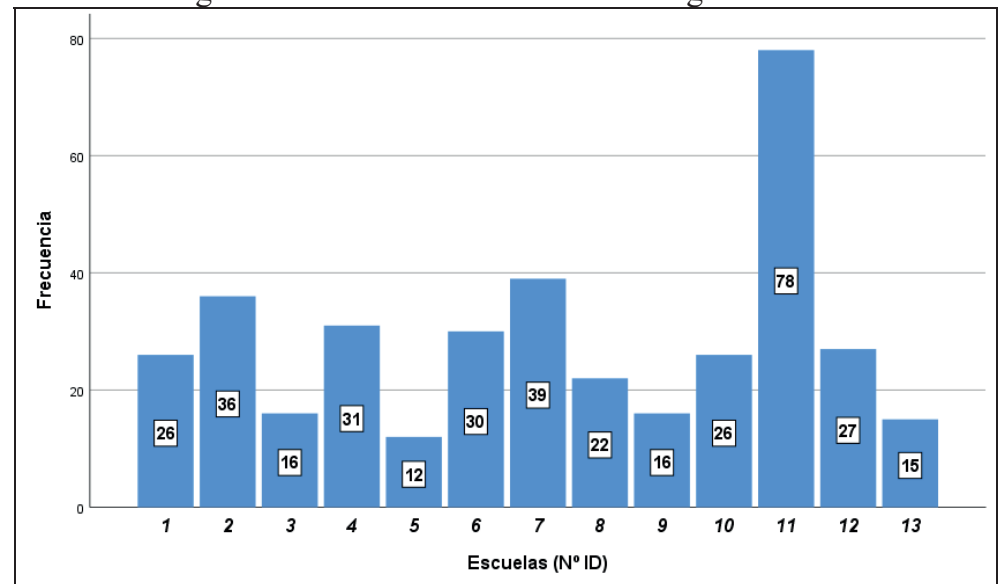

Fuente: Elaboración propia mediante IBM SPSS Statistics 25

Figura 2. Distribución de la Edad en la muestra de estudio $(\mathrm{N}=156)$

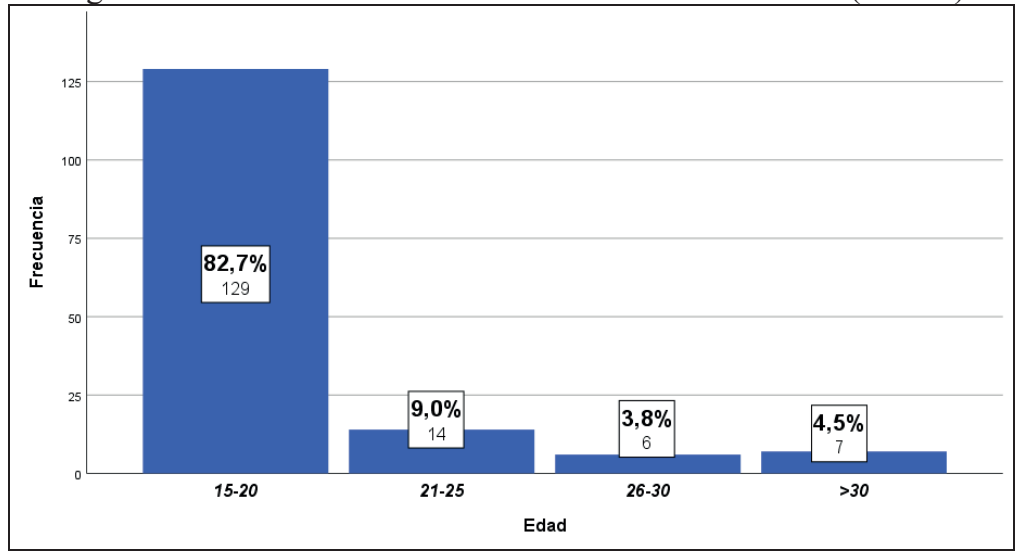

\section{Resultados del análisis inferencial comparativo}

\subsection{Factores intrínsecos del alumno}

El genero del potencial heredero es una de las primeras identidades individuales que debemos considerar. Existe una relación estadísticamente significativa $(\mathrm{p}<.05)$ entre la intención de seguir con la EAF y el sexo (efecto moderado-leve del 3.6\%) tal que podemos admitir que en las respuestas recibidas, esta intención es superior entre los hombres que entre las mujeres $(89.9 \%$ vs $62.5 \%)$.

Pero, en cambio, no existe ninguna relación estadísticamente significativa ( $p>.05)$ con la edad (efecto casi nulo: $<1 \%$ ). Por lo que no existe evidencia alguna de que la 
intención sucesoria se vincule a la edad del estudiante, estando esta intención siempre por encima del 85.7\%., analizamos otras posibles variables que pueden actuar como factores que influyen en la decisión de los alumnos de las Escuelas Agrarias de continuar con la EAF.

Tabla 1. Análisis inferencial comparativo. Intención sucesoria de EAF, en función del Sexo y de la Edad del encuestado. $(\mathrm{N}=156)$

\begin{tabular}{|c|c|c|c|c|c|c|c|}
\hline \multirow{3}{*}{$\begin{array}{l}\text { FACTOR } \\
\text { SEXO }\end{array}$} & \multirow[b]{2}{*}{ / Categorias } & \multirow{2}{*}{$\begin{array}{l}\text { Muestra total } \\
(\mathbf{N}=156)\end{array}$} & \multicolumn{2}{|c|}{ Intención sucesoria (\%) } & \multicolumn{2}{|c|}{$\begin{array}{l}\text { Test Chi- } \\
\text { cuadrado }\end{array}$} & \multirow{2}{*}{$\begin{array}{r}\text { Tamaño } \\
\text { del efecto } \\
R^{2}\end{array}$} \\
\hline & & & $\begin{array}{c}\mathbf{S i ́} \\
(88.5 \%)\end{array}$ & $\begin{array}{c}\text { NO } \\
(11.5 \%)\end{array}$ & Valor & $\begin{array}{c}P \\
\text { valor }\end{array}$ & \\
\hline & & & & & $5.57 *$ & .018 & .036 \\
\hline \multirow{7}{*}{$E D A D$} & Hombre & $(\mathrm{n}=148)$ & $89.9 \%$ & $10.1 \%$ & \multirow{7}{*}{$1.19^{\mathrm{NS}}$} & \multirow{7}{*}{.754} & \multirow{7}{*}{.008} \\
\hline & Mujer & $(\mathrm{n}=8)$ & $62.5 \%$ & $37.5 \%$ & & & \\
\hline & & & & & & & \\
\hline & $15-20$ años & $(\mathrm{n}=129)$ & $87.6 \%$ & $12.4 \%$ & & & \\
\hline & $21-25$ años & $(n=14)$ & $92.9 \%$ & $7.1 \%$ & & & \\
\hline & $26-30$ años & $(n=6)$ & $100 \%$ & $0.0 \%$ & & & \\
\hline & $=>31$ años & $(n=7)$ & $85.7 \%$ & $14.3 \%$ & & & \\
\hline N.S. $=\mathrm{NC}$ & significativo & ificativo & & & & & \\
\hline
\end{tabular}

El interés propio del sucesor potencial por gestionar su explotación agrícola es un factor de alta importancia. Según nuestros resultados es altamente significativo $(\mathrm{p}<.001)$ y con un efecto grande $(20.1 \%)$, de manera que entre los estudiantes con interés alto o muy alto en la EAF la intención sucesoria supera el 90\% (92.6\% y 100\% concretamente); en tanto que, entre el resto de los alumnos, la intención de seguir con la EAF es apenas del $50 \%$. Lo cual está de acuerdo con los resultados obtenidos por Morais, al afirmar que los sucesores potenciales que realizan estudios en agricultura se dan cuenta de su capacidad para controlar la empresa familiar rural, su deseo de trabajar en el sector agrícola aumenta y su intención de administrar la finca también aumenta (Morais et al., 2018).

La consideración acerca de la viabilidad de su propio futuro profesional también es un factor muy significativo $(\mathrm{p}<.01)$ aunque con menor efecto $(7.6 \%)$ pero aun notable. Los datos nos indican que la intención sucesoria en su EAF, es muy alta entre los que ven su futuro viable (96\%) o muy viable (97\%). Mientras que es mucho menor en el resto de los alumnos disminuyendo a medida que ven su futuro profesional menos viable $(81.5 \%$ y $73.7 \%)$.

La intención de crear su propio negocio también es un factor explicativo estadísticamente significativo $(\mathrm{p}<.05)$ y aún con efecto notable $(7.1 \%)$ : el $100 \%$ de los alumnos que ya tienen creado su propio negocio, van a seguir con la EAF y el $92.6 \%$ de los que tienen intención de crearlo, también quieren seguir con la EAF. Por el contrario, solo el $69.6 \%$ de los que no tiene intención de crear su negocio propio responde que seguirá con la EAF.

Finalmente, ni la variable del interés de los hermanos en continuar con la EAF, ni el rendimiento académico del propio estudiante, han aparecido como factores que explican la intención sucesoria propia del alumno, al no tener significación ( $\mathrm{p}>.05)$. Junto a ellos, tampoco son factores explicativos $(\mathrm{p}>.05)$ de la decisión del participante encuestado: ni el hecho de tener hermanos o no, ni el hecho de ser primogénito o no. 
Tabla 2. Análisis inferencial comparativo. Intención sucesoria de EAF, en función de los Factores Intrínsecos al encuestado. $(\mathrm{N}=156)$.

\begin{tabular}{|c|c|c|c|c|c|c|}
\hline \multirow{3}{*}{$\begin{array}{l}\text { FACTOR / Categorias } \\
\text { VIABILIDAD DEL FUTURO } \\
\text { PROFESIONAL }\end{array}$} & \multirow{2}{*}{$\begin{array}{c}\text { Muestra } \\
\text { total } \\
(\mathrm{N}=156)\end{array}$} & \multicolumn{2}{|c|}{ Intención sucesoria (\%) } & \multicolumn{2}{|c|}{ Test Chi-cuadrado } & \multirow{2}{*}{$\begin{array}{c}\text { Tamaño } \\
\text { del efecto } \\
\mathbf{R}^{2}\end{array}$} \\
\hline & & $\begin{array}{c}\mathbf{S I ́} \\
(88.5 \%)\end{array}$ & $\begin{array}{c}\text { NO } \\
(11.5 \%)\end{array}$ & Valor & Pvalor & \\
\hline & & & & $11.77 * *$ & .008 & .076 \\
\hline Muy probable viabilidad & $(n=33)$ & $97.0 \%$ & $3.0 \%$ & & & \\
\hline Probable viabilidad & $(\mathrm{n}=50)$ & $96.0 \%$ & $4.0 \%$ & & & \\
\hline No sabe & $(\mathrm{n}=54)$ & $81.5 \%$ & $18.5 \%$ & & & \\
\hline Poco/Nada probable viabilidad & $(\mathrm{n}=19)$ & $73.7 \%$ & $26.3 \%$ & & & \\
\hline INTENCIÓN CREAR PROPIO NEGOCIO & & & & $11.16 *$ & .011 & .071 \\
\hline Sí tiene intención & $(\mathrm{n}=81)$ & $92.6 \%$ & $7.4 \%$ & & & \\
\hline Si, ya lo ha hecho & $(n=13)$ & $100 \%$ & $0.0 \%$ & & & \\
\hline No sabe & $(n=39)$ & $87.2 \%$ & $12.8 \%$ & & & \\
\hline No tiene intención & $(n=23)$ & $69.6 \%$ & $30.4 \%$ & & & \\
\hline RESULTADOS ACADÉMICOS PROPIOS & & & & $2.35^{\mathrm{NS}}$ & .401 & .017 \\
\hline Excelentes & $(\mathrm{n}=16)$ & $87.5 \%$ & $12.5 \%$ & & & \\
\hline Notable & $(n=67)$ & $92.5 \%$ & $7.5 \%$ & & & \\
\hline Aprobado/Suspenso & $(n=69)$ & $84.9 \%$ & $15.1 \%$ & & & \\
\hline INTERÉS PROPIO EN LA EAF & & & & $32.16 * *$ & .000 & .201 \\
\hline Muy alto & $(\mathrm{n}=69)$ & $97.1 \%$ & $2.9 \%$ & & & \\
\hline Alto & $(n=69)$ & $89.9 \%$ & $10.1 \%$ & & & \\
\hline Neutro/Bajo/Ninguno & $(n=18)$ & $50.0 \%$ & $50.0 \%$ & & & \\
\hline TIENE HERMANOS & & & & $0.73^{\mathrm{NS}}$ & .393 & .005 \\
\hline$S i$ & $(\mathrm{n}=132)$ & $89.4 \%$ & $10.6 \%$ & & & \\
\hline No & $(n=24)$ & $83.3 \%$ & $16.7 \%$ & & & \\
\hline ES PRIMOGÉNITO & & & & $0.77^{\mathrm{NS}}$ & .381 & .005 \\
\hline$S i$ & $(\mathrm{n}=89)$ & $86.5 \%$ & $13.5 \%$ & & & \\
\hline No & $(n=67)$ & $91.0 \%$ & $9.0 \%$ & & & \\
\hline $\begin{array}{l}\text { INTERÉS RESTO HERMANOS EN LA } \\
\text { EAF }\end{array}$ & & & & $4.29^{\mathrm{NS}}$ & .369 & .032 \\
\hline Muy alto & $(\mathrm{n}=11)$ & $100 \%$ & $0.0 \%$ & & & \\
\hline Alto & $(n=25)$ & $96.0 \%$ & $4.0 \%$ & & & \\
\hline Neutro & $(n=31)$ & $83.9 \%$ & $16.1 \%$ & & & \\
\hline Bajo & $(n=41)$ & $85.4 \%$ & $14.6 \%$ & & & \\
\hline Muy bajo & $(n=24)$ & $91.7 \%$ & $8.3 \%$ & & & \\
\hline
\end{tabular}

\subsection{Factores relativos al titular de las EAF}

En este caso los resultados que hemos obtenido (Tabla 3) nos llevan a concluir que, con una excepción, estas variables que responden a preguntas relativas al titular de EAF, no se constituyen como factores explicativos de la intención sucesoria del alumno encuestado ( $\mathrm{p}>.05$ y tamaños de efecto muy bajos). Por tanto: quién sea el titular, el tiempo de trabajo que dedica, la opinión sobre la vida agraria y la intención de traspaso que tiene el titular, no están relacionados con que el alumno quiera seguir con la EAF.

La excepción la hemos encontrado en el hecho concreto de que el titular haya manifestado su intención concreta de traspaso de la EAF, donde aparece una alta significación $(\mathrm{p}<.001)$ y un efecto ya muy grande $($ del $27.1 \%)$. Este factor se convierte en explicativo porque los datos revelan que un $98.3 \%$ de aquellos que expresamente nombrados sucesores por el titular tienen la intención de aceptar y seguir con la EAF; así como el 100\% de los que marcaron "otras opciones" en la respuesta a la cuestión de esta variable. Por el contrario, que el alumno quiera seguir con la EAF desciende mucho cuando la intención manifiesta del titular es traspasar el negocio a otros hermanos $(66.7 \%)$ o a otros familiares $(57.1 \%)$. 
Tabla 3. Análisis inferencial comparativo. Intención sucesoria de EAF, en función de los Factores relativos al Titular de la EAF. $(\mathrm{N}=156)$.

\begin{tabular}{|c|c|c|c|c|c|c|}
\hline \multirow{3}{*}{$\begin{array}{l}\text { FACTOR / Categorias } \\
\text { TITULAR DE LAS EAF }\end{array}$} & \multirow{2}{*}{$\begin{array}{c}\text { Muestra } \\
\text { total } \\
(\mathrm{N}=156)\end{array}$} & \multicolumn{2}{|c|}{ Intención sucesoria (\%) } & \multicolumn{2}{|c|}{ Test Chi-cuadrado } & \multirow{2}{*}{$\begin{array}{c}\text { Tamaño } \\
\text { del efecto } \\
\mathbf{R}^{2}\end{array}$} \\
\hline & & $\begin{array}{c}\mathbf{S I} \\
(88.5 \%)\end{array}$ & $\begin{array}{c}\text { NO } \\
(11.5 \%)\end{array}$ & Valor & Pvalor & \\
\hline & & & & $2.63^{\mathrm{NS}}$ & .453 & .016 \\
\hline Padre (solo) & $(\mathrm{n}=85)$ & $89.4 \%$ & $10.6 \%$ & & & \\
\hline Madre (sola) & $(\mathrm{n}=10)$ & $100 \%$ & $0.0 \%$ & & & \\
\hline Padre y Madre & $(n=27)$ & $88.9 \%$ & $11.1 \%$ & & & \\
\hline Otros familiares & $(n=34)$ & $82.4 \%$ & $17.6 \%$ & & & \\
\hline TIEMPO TRABAJO DEL TITULAR & & & & $0.28^{\mathrm{NS}}$ & .600 & .002 \\
\hline A tiempo completo & $(\mathrm{n}=87)$ & $89.7 \%$ & $10.3 \%$ & & & \\
\hline A tiempo parcial & $(n=69)$ & $87.0 \%$ & $13.0 \%$ & & & \\
\hline OPINIÓN SOBRE VIDA AGRARIA & & & & $6.22 \mathrm{NS}$ & .102 & .041 \\
\hline Muy satisfecho/a & $(n=55)$ & $96.4 \%$ & $3.6 \%$ & & & \\
\hline Satisfecho/a & $(n=64)$ & $82.8 \%$ & $17.2 \%$ & & & \\
\hline Neutro & $(n=26)$ & $92.3 \%$ & $7.7 \%$ & & & \\
\hline Poco/Nada satisfecho/a & $(n=6)$ & $83.3 \%$ & $16.7 \%$ & & & \\
\hline INTENCIÓN DE TRASPASO & & & & $2.64^{\mathrm{NS}}$ & .267 & .017 \\
\hline$S i$ & $(\mathrm{n}=80)$ & $92.5 \%$ & $7.5 \%$ & & & \\
\hline No & $(n=56)$ & $83.9 \%$ & $16.1 \%$ & & & \\
\hline Otras opciones & $(n=20)$ & $85.0 \%$ & $15.0 \%$ & & & \\
\hline A QUIÉN TRASPASA $(N=80)$ & & & & -- & -- & -- \\
\hline Otro miembro de la familia & $(n=77)$ & $92.2 \%$ & $7.8 \%$ & & & \\
\hline Vienta & $(\mathrm{n}=1)$ & -- & -- & & & \\
\hline Otras opciones & $(n=2)$ & -- & -- & & & \\
\hline TRASPASO A UN FAMILIAR $(N=77)$ & & & & $20.90 * *$ & .000 & .271 \\
\hline Al encuestado & $(n=59)$ & $98.3 \%$ & $1.7 \%$ & & & \\
\hline A otros hermanos & $(n=6)$ & $66.7 \%$ & $33.3 \%$ & & & \\
\hline A otros familiares & $(\mathrm{n}=7)$ & $57.1 \%$ & $42.9 \%$ & & & \\
\hline Otras opciones & $(n=5)$ & $100 \%$ & $0.0 \%$ & & & \\
\hline N.S. $=$ NO significativo $\quad * *=$ Altamente significativo & & & & & & \\
\hline
\end{tabular}

\section{Relación entre la Intención sucesoria de la EAF y la SEW (Escala REI)}

Para establecer la relación entre intención sucesoria de la EAF y la SEW se ha empleado la versión reducida REI (Hauch et al., 2016) del cuestionario FIBER. Pretendemos evaluar el constructo teórico de la Riqueza Emocional con la validación empírica de la escala FIBER (Pearson y Lumpkin, 2011).

La escala FIBER en la versión REI consta de 16 ítems en formato Likert de 5 opciones, configurados sobre 3 dimensiones: (a) $\mathbf{R}$ - Renovación de los lazos familiares a través de la sucesión, 4 ítems; (b) Relación Emocional -E- de los miembros familiares, 6 ítems; y (c) I- Identificación de los miembros familiares con la empresa, otros 6 ítems. Para el presente análisis estadístico, y con la intención de que una mayor puntuación en la variable se interprete como un grado más alto (mayor dominio) en la dimensión evaluada, se han recodificado las respuestas de los encuestados en la escala: $1=$ nada de acuerdo, hasta $5=$ muy de acuerdo.

Resultados de la validación de la Escala REI en el contexto de EAF.

En primer lugar, se ha procedido a comprobar si es factible aplicar dentro del ámbito concreto de las EAF, esta escala creada inicialmente en un contexto amplio de empresas familiares. Luego, se procedió a revalidar la escala REI (Hauch et al., 2016) para la población de alumnos de Escuelas Agrarias que están vinculados a EAF. Se empleó la metodología del Análisis Factorial por Componentes Principales, para verificar la unidimensionalidad de cada conjunto de ítems con la dimensión a la que teóricamente pertenecen. 
Se obtuvieron tres dimensiones que agrupan un conjunto de ítems que miden las dotes afectivas básicas que una familia puede derivar del control de una empresa. También se estimó el grado de fiabilidad de cada una de estas dimensiones, mediante el Coeficiente "Alfa" de Cronbach, así como de cada uno de los ítems con el Índice de homogeneidad ítem-total.

- Dimensión R. Se refiere a la renovación de los lazos familiares en la sucesión, donde los ítems referidos a continuar con el legado y tradición familiar, valorar la inversión de la familia como de largo plazo, y donde la transferencia de la explotación agraria familiar a la próxima generación es un objetivo importante para los miembros de la familia.

Los valores medios de estos 4 ítems son elevados (entre 3.82 y 4.43 ; sobre 5 ), lo que indica que la posición de los estudiantes de nuestra muestra tiende claramente hacia el acuerdo a cada propuesta realizada para evaluar esta dimensión. Los valores de las comunalidades son óptimos en todos los ítems y las cargas factoriales son altas (entre .617 y .813). Estos datos demuestran la unidimensionalidad de estos ítems con respecto a la dimensión R. Por otro lado, los índices de fiabilidad de los ítems son suficientes (>.300) de manera que la fiabilidad de esta dimensión es buena (.664), (Tabla 4).

Tabla 4. Unidimensionalidad y Fiabilidad. Dimensión: R - Renovación de los lazos familiares a través de la sucesión; del REI. ( $\mathrm{N}=156)$

\begin{tabular}{|c|c|c|c|c|}
\hline \multirow{2}{*}{ ITEM } & \multirow{2}{*}{$\begin{array}{c}\text { Descriptivos } \\
\text { Media (D.E.) }\end{array}$} & \multicolumn{2}{|c|}{$\begin{array}{l}\text { Análisis Factorial por } \\
\text { C.P. }\end{array}$} & \multirow{2}{*}{ Fiabilidad } \\
\hline & & Comunalidad & $\begin{array}{c}\text { Carga } \\
\text { Factorial }\end{array}$ & \\
\hline$S E W-R 1$ & $4.43 \quad(0.72)$ & .381 & .617 & .359 \\
\hline$S E W-R 2$ & $3.82(0.94)$ & .442 & .665 & .409 \\
\hline$S E W-R 3$ & $4.05 \quad(1.17)$ & .536 & .732 & .474 \\
\hline$S E W-R 4$ & $4.17 \quad(0.93)$ & .661 & .813 & .574 \\
\hline Total & $16.47(2.69)$ & --- & $50.49 \%$ & .664 \\
\hline
\end{tabular}

- Dimensión E. Se refiere al apego emocional de los miembros de la familia donde se consideran si los lazos emocionales son fuertes o no, si esos lazos emocionales ayudan a tener un concepto positivo de cada uno de los miembros y si en la empresa los miembros de la familia se sienten afectuosos entre ellos.

De nuevo los valores medios de todos los ítems son altos (entre 4.11 y 4.33 ) indicando opiniones favorables hacia el contenido enunciado en cada uno de ellos. Los valores de las comunalidades son muy elevados y los pesos factoriales también (entre .730 y .825). Por tanto, queda demostrada la pertenencia unidimensional de estos 6 ítems a la dimensión E. Así mismo, los índices de fiabilidad de estos ítems son altos (>.600), lo que genera un coeficiente de fiabilidad en la dimensión elevado (.857), (Tabla 5). 
Tabla 5. Unidimensionalidad y Fiabilidad. Dimensión: E - Relación Emocional de los miembros familiares; del REI. ( $\mathrm{N}=156)$

\begin{tabular}{|c|c|c|c|c|}
\hline \multirow[b]{2}{*}{ ITEM } & \multirow{2}{*}{$\begin{array}{l}\text { Descriptivos } \\
\text { Media (D.E.) }\end{array}$} & \multicolumn{2}{|c|}{ Análisis Factorial por C.P. } & \multirow[b]{2}{*}{ Fiabilidad } \\
\hline & & Comunalidad & $\begin{array}{c}\text { Carga } \\
\text { Factorial }\end{array}$ & \\
\hline$S E W-E 1$ & $4.11 \quad(0.97)$ & .652 & .807 & .699 \\
\hline$S E W-E 2$ & $4.33(0.76)$ & .539 & .734 & .611 \\
\hline$S E W-E 3$ & $4.17 \quad(0.82)$ & .680 & .825 & .722 \\
\hline$S E W-E 4$ & $4.13(0.83)$ & .533 & .730 & .606 \\
\hline$S E W-E 5$ & $4.16(0.84)$ & .539 & .734 & .614 \\
\hline$S E W-E 6$ & $4.31 \quad(0.86)$ & .567 & .753 & .629 \\
\hline Total & $25.22(3.89)$ & --- & $58.49 \%$ & .857 \\
\hline
\end{tabular}

- Dimensión I. Se refiere a la identificación de los miembros de la familia con la explotación agraria, donde los miembros tienen un fuerte sentido de pertenencia y un gran significado personal hacia la explotación hasta el punto de sentirse orgullosos de decir a los demás que pertenecen a la explotación familiar.

Esta dimensión ha alcanzado significación $(\mathrm{p}<.05)$ lo que junto a un efecto moderado nos permite admitir que tenemos evidencia estadística que relaciona la identificación del estudiante con la empresa familiar por sus valores emocionales (Tabla 6). Las puntuaciones medias indican una cierta linealidad, siendo más alta la puntuación en la identificación de los miembros familiares con la empresa, cuanto más posible se considera la viabilidad del negocio.

Tabla 6: Análisis inferencial comparativo. Diferencias en la SEW en función de la viabilidad profesional en la agricultura del encuestado. $(\mathrm{N}=156)$.

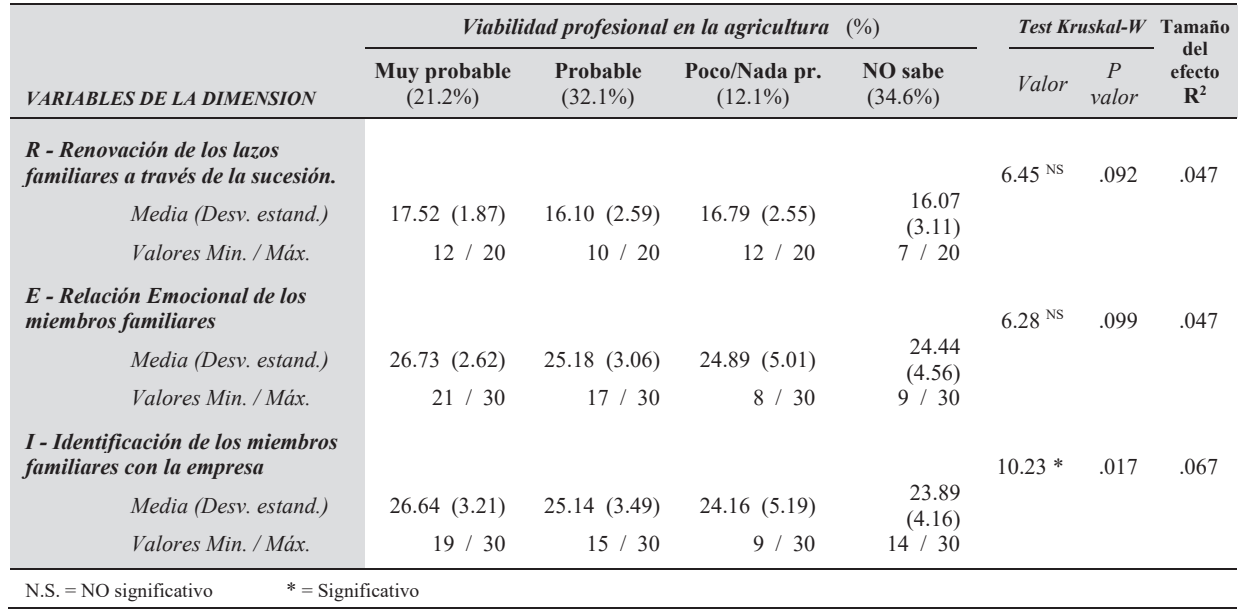

\section{Discusión y conclusiones}

Este trabajo analiza la intención sucesoria de los estudiantes de escuelas agrarias que forman parte de una explotación agraria familiar. Para ello se ha acudido a las 13 Escuelas Agrarias de Catalunya, obteniendo respuesta de 374 estudiantes, sobre una población de 700. De las respuestas obtenidas, muy cerca de la mitad (161) han respondido que forman parte de una Explotación Agraria Familiar. 
Acudir a las escuelas agrarias nos ha permitido tener acceso a una población que, de otra manera, se encuentra muy diseminada y que genera una dificultad añadida a la obtención de datos. Otro elemento a tener en cuenta es la obtención de datos de los actores a analizar (jóvenes de explotaciones agrarias familiares y que se están formando en escuelas agrarias), siendo la población objeto de estudio quién proporciona la respuesta (cuestión demandada en la literatura de la economía y la sociología agraria).

Los resultados obtenidos a partir del análisis inferencial comparativo y en relación con los factores intrínsecos del alumno nos indican que el género, el interés propio, la propia creencia acerca de la viabilidad de su futuro profesional en el sector agrario, así como la intención de crear su propio negocio son elementos con una alta influencia en la intención sucesoria de estos estudiantes y que influyen en la misma. Por otra parte, ni la edad, ni la primogenitura, ni el interés demostrado por los hermanos tiene ninguna influencia en la intención sucesoria de estos estudiantes.

En lo referente a las características del titular de la explotación agraria, el único elemento significativo al respecto de la influencia en la intención sucesoria de estos estudiantes se da en el hecho que el titular haya manifestado su voluntad de traspasar la explotación y que este traspaso recaiga en el encuestado; ni el género del titular, ni el tipo de trabajo en la explotación agraria familiar, ni la opinión del mismo al respecto de su dedicación al sector agrario, ejercen ningún tipo de influencia.

Finalmente, la validación de la escala REI de análisis del nivel de riqueza socioemocional (SEW) en la figura de los potenciales y/o futuros sucesores nos permite ampliar y aplicar esta escala más allá de los actores a quién se les había aplicado para obtener el nivel de SEW en las empresas familiares, a los titulares de estas. Esta validación nos permite incorporar esta escala como instrumento de análisis de un actor clave para la continuidad y el futuro de las explotaciones agrarias familiares, como es el futuro sucesor, a partir de su intención sucesoria. Se permite incorporar al análisis de las explotaciones agrarias familiares la mirada, la intención y la visión del joven futuro sucesor de las explotaciones agrarias familiares.

\section{Referencias}

Alsos, A; Carter, S. y Ljunggren, E. (2011). "The Handbook of Research on Entrepreneurship in Agriculture and Rural Development". Edward Elgar Publishing, Uk.

Berrone, P.; Cruz, C. y Gómez-Mejia, L.R. (2012). "Socio-emotional wealth in family firms: theoretical dimensions, assessment approaches, and agenda for future research". Family Business Review, 25(3), 258-279.

Cavicchioli, D.; Bertoni, D.; Tesser, F. y Frisio, D.G. (2015). "What Factors Encourage Intrafamily Farm Succession in Mountain Areas?" Mountain Research and Development, 35(2), 152-160.

Chiswell, H.M. (2014). "The importance of next generation farmers: a conceptual framework to bring the potential successor into focus". Geography Compass 8 (5), 300312.

Corsi, A. (2004). "Intra-family succession in Italian farms". Paper prepared for presentation at the SFER Conference: Les mutations de la famille agricole: Consequences pour les politiques publiques, Paris, France, April 2004. 
Darnhofer, I.; Fairweather, J. y Moller, H. (2010). "Assessing a farm's sustainability: Insights from resilience thinking". International Journal of Agricultural Sustainability, 8(3), 186-198.

Dinis, I. (2019). "The concept of Family Farming in the Portuguese Political Discourse". Social Sciences MDPI. 8(7), 1-15.

Gallizo, J.L.; Mar, C.; Moreno, J. y Salvador, M. (2017). "Family business and valueadded distribution: a socioemotional wealth approach". Academia. Revista Latinoamericana de Administración, 30(1), 2-22.

Glover, J. (2014). "Gender, power and succession in family farm business". International Journal of Gender and Entrepreneurship, 6 (3), 276-295.

Gómez-Mejía, L.R.; Haynes, K.T.; Núñez-Nickel, M.; Jacobson, K.J.L.; y MoyanoFuentes, H. (2007). "Socioemotional wealth and business risk in family-controlled firms: Evidence from Spanish olive oil mills". Administrative Science Quarterly, 52(1), 106-137.

Gomez-Mejia, L. R., Makri, M., \& Larraza Kintana, M. (2010). Diversification decisions in family-controlled firms. Journal of Management Studies, 47, 223-252.

Gómez-Mejía, L.R.; Cruz, C.; Berrone, P. y De Castro, J. (2011). "The bind that ties: Socioemotional wealth preservation in family firms". Academy of Management Annals, 5(1), 653-707.

Hauck, J.; Suess-Reyes, J.; Beck, S.; Prügl, R. y Frank, F. (2016). "Measuring socioemotional wealth in family-owned and -managed firms: A validation and short form of the FIBER Scale”. Journal of Family Business Strategy, 7(3), 133-148.

Hennessey, T. y Rehmann, T. (2007). "An Investigation into Factors Affecting the Occupational Choices of Nominated Farm Heirs in Ireland". Journal of Agricultural Economics, 58(1), 61-75.

Iinwood, S.M. y Sharp, J.S. (2012). "Farm persistence and adaptation at the rural-urban interface: Succession and farm adjustment”. Journal of Rural Studies, 28(1), 107-117.

Jenkins, R. (1996) Social identity (London: Routledge).

Keating, N. y Munro, B. (1989). "Transferring the family farm: Process and implications". Family Relations, 38, 215-218.

Lange A.; Piorr A.; Siebert, R. y Zasada, I. (2013). "Spatial differentiation of farm diversification: How 32 rural attractiveness and vicinity to cities determine farm households' response to the CAP". Land Use Policy, 31, 136-144.

Leonard, B.; Kinsella, A.; O'Donoghue, C.; Farrell, M. y Mahon, M. (2017). "Policy drivers of farm succession and inheritance". Land Use Policy, 61, 147-159.

Lobley, M. y Potter, C.A. (2004). "Agricultural change and restructuring: recent evidence from a survey of agricultural households in England". Journal of Rural Studies, 20, 499-510.

Lobley, M., Baker, J. y Whitehead, I. (2010). "Farm succession and retirement: Some international comparisons". Journal of Agriculture, Food Systems and Community Development, I(1), 49-64. 
Mann S., Mittenzwei K., Hasselmann F. 2013. The importance of succession on business growth: A case study of family farms in Switzerland and Norway. Yearbook of Socioeconomics in Agriculture, 2013: 109-137.

Morais, M.; Borges, J.A.R. y Binotto, E. (2018). "Using the reasoned action approach to understand Brazilian successors' intention to take over the farm". Land Use Policy, 71, $445-452$.

Naldi, L.; Cennamo, C.; Corbetta, G. y Gómez-Mejía, L.R. (2013). "Preserving socioemotional wealth in family firms: Asset or liability? The moderating role of business context". Entrepreneurship Theory and Practice, 37, 1341-1360.

Pearson, W.A. y Lumpkin, T. (2011). "Mesurement in Family Business Research: How do we measure up?". Family Business Review, 24(4), 287-291

Rosenzweig, M.R. y Wolpin, K.I. (1985). "Specific Experience, Household Structure, and Intergenerational Transfers: Farm Family Land and Labor Arrangements in Developing Countries". The Quarterly Journal of Economics, 100(supl), 961-987.

Schulze, W.S. y Kellermanns, F.W. (2015). "Reifying Socioemotional Wealth". Entrepreneurship Theory and Practice, May, 447-459.

Suess-Reyes, J. y Fuetsch, E. (2016). "The future of family farming: A literature review on innovative, sustainable and succession-oriented strategies". Journal of Rural Studies, 47(A), 117-140.

Sumane, S.; Kunda, I.; Knickel, K. y Strauss, A. (2018). "Local and farmers' knowledge matters! How integrating informal and formal knowledge enhances sustainable and resilient agriculture", Journal of Rural Studies, 59, 232-241.

Unión Europea, Europea-International" (www.europea.org)

Zagata, L. y Sutherland, L.A. (2015). "Deconstructing the "young farmer problem in Europe': towards a research agenda". Journal Rural Studies, 38 (April 2015), 39-51. 